\title{
Using Acupressure to Modify Alertness in the Classroom: A Single-Blinded, Randomized, Cross-Over Trial
}

\author{
RICHARD E. HARRIS, Ph.D., ${ }^{1,2}$ JOANNE JETER, ${ }^{2}$ M.D., PAUL CHAN, M.D., ${ }^{2}$ \\ PETER HIGGINS, M.D. Ph.D., ${ }^{2}$ FENG-MING KONG, M.D., ${ }^{2}$ REZA FAZEL, M.D., ${ }^{2}$ \\ CANDACE BRAMSON, M.D., ${ }^{2}$ BRENDA GILLESPIE, Ph.D., ${ }^{2}$ \\ AND COHORT 11 OF THE UNIVERSITY OF MICHIGAN CLINICAL RESEARCH DESIGN \\ AND STATISTICAL ANALYSIS PROGRAM ${ }^{2 *}$
}

\begin{abstract}
Background: Previous reports have suggested that acupressure is effective in reducing pain and improving sleep quality; however, its effects on alertness have not been characterized.

Objectives: The aim of this study was to determine whether two different acupressure treatments have opposing effects on alertness in a full-day classroom setting.

Design: This was a cross-over (two-treatments; three periods), single-blinded, randomized trial.

Setting: The University of Michigan School of Public Health was the setting.

Subjects: Students attending a course in clinical research design and statistical analysis at the University of Michigan participated in the study.

Interventions and outcome measures: Blinded subjects were randomized to two acupressure treatment sequences: stimulation-relaxation-relaxation or relaxation-stimulation-stimulation. Acupressure treatments were self administered over 3 consecutive days. Pre- and post-treatment alertness scores were assessed each day using the Stanford Sleepiness Scale (SSS). Changes in the SSS score (afternoon - morning) were analyzed using a mixed regression model of fixed and random effects. Important factors that were expected to affect alertness, such as caffeine and previous night's sleep, were also assessed.

Results: Baseline characteristics and protocol compliance were similar between the two sequences. Stimulation acupressure treatment yielded a 0.56-point greater difference in score on the SSS, corresponding to less fatigue, compared to the relaxation acupressure treatment $(p=0.019)$. Day of study $(p=0.004)$ and hours of overnight sleep $(p=0.042)$ also significantly affected the change in SSS scores. Incorporating participants' beliefs as to which treatment they received did not significantly alter the observed treatment effect.

Conclusions: Acupressure at stimulation and relaxation points has differential effects on alertness in a classroom setting. Further research is necessary to confirm these findings and to determine whether stimulation and relaxation acupressure are equally effective in influencing alertness.

\footnotetext{
${ }^{1}$ Department of Internal Medicine, Division of Rheumatology, University of Michigan, Ann Arbor, MI.

${ }^{2}$ Course on Clinical Research Design and Statistical Analysis, University of Michigan, School of Public Health. Ann Arbor MI.

* Members of the University of Michigan Clinical Research Design and Statistical Analysis Program Cohort 11 are: Khawaja Afzal Ammar, Michelle Anderson, Ghassan Bachuwa, Adnan Beg, Devin Brown, Linda Brubaker, Steven Bruch, Giani Cazan-London, Bobby Clark, Paul Cronin, Brian DeSmet, Gary Hamman, Brent Hollenbeck, Jaquelyn Hunt, Susan Johnson, Sucheta Joshi, Liise Kayler, Aine Kelly, Kim Kenton, Wendy Marder, Mary Marzec, Gisele Neiva, Amanda Peltier, Panduranga Rao, Erik Roys, Joel Rubenstein, Jonathan Segal, T.P. Singh, Maran Thamilarasan, Courtney Thornburg, Kevin Townsend, Robert Welch, Jinping Xu, and Najeeb Zoubi.
} 


\section{INTRODUCTION}

A lertness in the workplace has generated considerable attention over the last two decades. Of particular interest is the effect of fatigue in medical trainees, which has recently prompted a significant change in national regulations regarding trainee work hours in the United States. ${ }^{1}$ Although studies confirm the negative effects of decreased workplace alertness in a variety of workers, few investigations offer interventions to counteract the effects of fatigue. ${ }^{2-6}$

To date, no reports of acupuncture or acupressure treatments to increase alertness are available. However, these modalities have been evaluated as treatments to improve the quality of sleep and to reduce fatigue. Success rates as high as $90 \%$ have been recorded for use of acupuncture in the treatment of insomnia. ${ }^{7}$ Tsay et al. examined the effects of acupressure on sleep and fatigue in Taiwanese patients with end-stage renal disease. In one trial, subjects were randomized to acupressure three times per week for 4 weeks, sham acupressure with the same schedule, or control groups without acupressure. ${ }^{8,9}$ The acupressure group reported a significant decrease in wake time (amount of time spent awake during "sleeping" hours) as well as an improved score on the Pittsburgh Sleep Quality Index compared to the control group; however, no differences were observed between sham and real acupressure. Similar results were obtained from another study by Tsay assessing changes in fatigue using a visual analog scale. ${ }^{10}$ Significant improvements in fatigue were found when comparing the control group to the acupressure and sham acupressure groups, yet no differences were observed between sham and real acupressure treatments. These results suggest that for treating fatigue, sham acupressure may not be an inactive control condition; alternatively, real acupressure may have no significant benefit versus placebo.

Therefore, a single-blind, randomized, cross-over study was conducted to determine whether acupressure has a significant effect on alertness in a population of healthy medical professionals in a prolonged lecture situation. The study used two active treatments, a relaxation treatment and a stimulation treatment, in a cross-over design in which all participants received both treatments on different days. This design avoids the problem of potentially active sham acupressure treatments and maximizes the likelihood of observing an effect of acupressure on alertness, as the two treatments presumably have opposite effects.

\section{MATERIALS AND METHODS}

\section{Study objective}

The objective of this study was to evaluate the effect of acupressure on the mental alertness of subjects participating in a 3-day lecture environment. The hypothesis was that participants performing stimulatory acupressure would have smaller increases in fatigue from their morning baseline measurements to their end-of-day assessments, compared to days when the same individuals performed a relaxation acupressure technique. The study protocol was reviewed and approved by the University of Michigan Institutional Review Board, and informed consent was obtained from all subjects.

\section{Study population}

The study population consisted of 39 subjects enrolled at the University of Michigan School of Public Health course on Clinical Research Design and Statistical Analysis (CRDSA). Inclusion criteria were: (1) $\geq 18$ years of age; (2) member of the CRDSA cohort XI; and (3) present during the May 2004 CRDSA session. Exclusion criterion was prior experience with acupuncture or acupressure. Participants included physicians, nurses, dentists, pharmacists, and professionals from the health care industry.

\section{Interventions}

Acupressure regimens promoting mental stimulation or relaxation were used in a cross-over design with subjects randomized to either Sequence I (stimulation-relaxation-relaxation) or Sequence II (relaxation-stimulation-stimulation). Each regimen was taught to all study participants by two members of the class who were previously trained in acupressure (one with 3 years and the other with 5 years of experience). The instruction and supervision of acupressure was carried out in separate rooms, on 3 consecutive days during the lunch hour. Each regimen consisted of a 15minute self-administered session of acupressure at either five stimulatory or five relaxation points ( 3 minutes each). The stimulatory point formula consisted of Si Shen Chong and bilateral-LI 4, St 36, K 1, and UB 10. These points were chosen based on their ability to reduce fatigue and sleepiness in Traditional Chinese Medical (TCM) theory. ${ }^{11}$ The relaxation point formula was selected based on treatment of insomnia in TCM theory and contained Yin Tang and bilateral Anmian, Ht 7, Liv 3, Sp 6. ${ }^{11}$ These point formulas were refined by two additional acupuncturists outside of the study but trained in TCM theory ( 5 years experience each) and the consultation of additional TCM texts by the participating acupuncturists. ${ }^{12,13}$ The stimulation and relaxation points are illustrated in Figure 1.

All treatments were performed in cranio-caudal sequence. Acupressure was self administered at Si Shen Chong and Anmian by light tapping with the fingers at a frequency of $2 \mathrm{~Hz}$. All other points were stimulated using either the thumb or forefingers to massage in both clockwise and counterclockwise directions. The style of acupressure administered in this investigation was highly simplified as to allow for the training of participants and to allow for the limited study time period. The instructors demonstrated the intensity of pressure required at each 


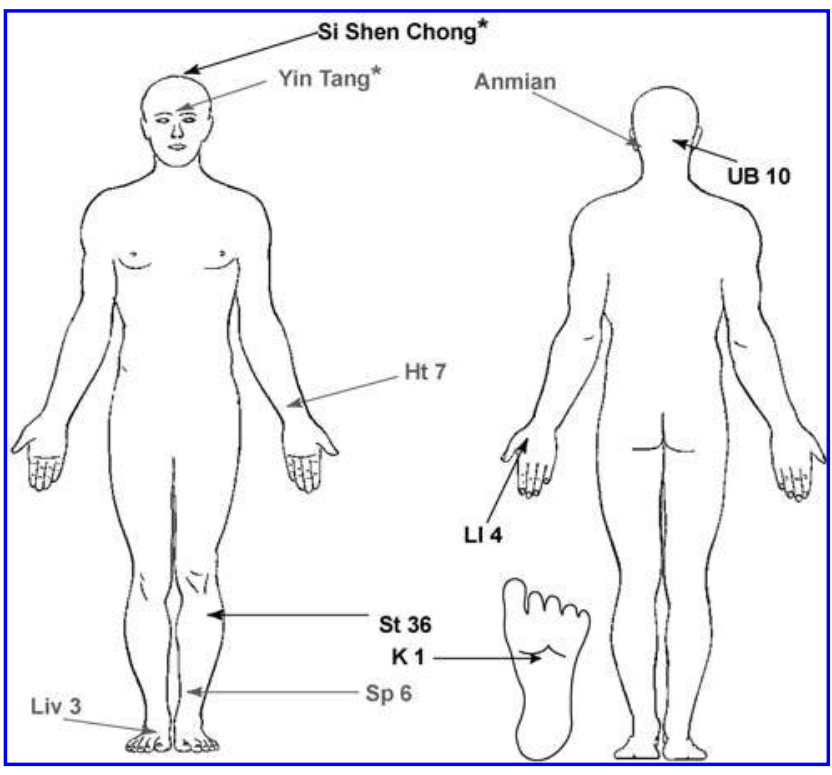

FIG. 1. Body maps of stimulation (black) and relaxation (gray) acupressure locations. *, Pressure applied unilaterally. All other points were bilaterally stimulated.

point once on all subjects to ensure that adequate pressure was applied.

\section{Randomization}

The 39 eligible subjects were randomized to one of two sequences using the pseudo-random number function of a Texas Instruments TI- 83 calculator (Dallas, TX). Those in sequence I self administered acupressure in the sequence: stimulation (day 1)-relaxation (day 2)-relaxation (day 3). Those in sequence II self administered acupressure in the sequence: relaxation (day 1)-stimulation (day 2)-stimulation (day 3).

\section{Masking}

All subjects were masked as to whether the regimen that they performed each day was the stimulation or the relaxation formula. To assess the success of masking, subjects were asked each day to guess their treatment assignment after administering acupressure. The principal investigators, the randomization team, the data collection team, and the data analysts were also all masked as to the identity of the interventions.

\section{Outcomes}

The primary outcome was the difference between afternoon and morning scores on the Stanford Sleepiness Scale (SSS) (afternoon score - morning score). ${ }^{14}$ The SSS is an 8-point ordinal scale that assesses subjective sleepiness. The SSS was administered 2 hours before and 3 hours after each acupressure regimen. The 3 -hour endpoint was chosen be- cause this was the end of the class period and students were most likely to be fatigued. A daily pretreatment questionnaire included morning sleepiness level as measured by the SSS, hours of sleep the previous night, ounces of caffeinated coffee, ounces of caffeinated tea, ounces of caffeinated cola, alcohol in the last 24 hours (yes/no), and medication use in the last 24 hours (yes/no), the occurrence of any unusual or upsetting events (yes/no), and presence of anxiety that day (yes/no). A compliance and masking questionnaire was also administered each day. The compliance questionnaire contained a Visual Analog Scale asking: "How confident are you that you have self-administered the acupressure treatment appropriately?" with anchors "not confident" and "very confident." After the third and final acupressure period, information was collected about adverse events during the study.

\section{Statistical methods}

Data from self-report forms were entered into a Microsoft Access database (Microsoft Corp., Redmond, WA) using a two-person, dictation-entry method. To assess covariate balance by treatment, baseline characteristics were compared between the two treatment sequences. To assess changes over the study period, the same covariates were compared over the 3 study days. Nine subjects with missing data were asked to provide the data retrospectively.

A model was developed using the change in the SSS score from morning to afternoon as the dependent variable, and all other baseline covariates as independent variables. Data were analyzed using the PROC MIXED procedure of SAS version 8.2 (SAS Institute, Cary, NC). This model incorporated the fixed effects of sequence (I or II), period (day 1, 2 , or 3), treatment (relaxation or stimulation), and other covariates, as well as the random effects of subject within sequence. Only those variables with values of $p<0.20$ were retained in the model. A significance level of $\alpha=0.05$ was used in interpretation of the final analysis. Least-square means of the primary outcome were contrasted for the two treatments.

Masking was evaluated by a $2 \times 2$ contingency table analysis of which treatments subjects believed that they had received and their actual treatments. The effect of masking was further evaluated by adding each subject's assessment of the treatment assignment into the model as a covariate.

After inspection of the crude data, one subject from sequence II was identified as an influential outlier. This participant displayed extreme fatigue and sleepiness during the study because of prolonged sleep deprivation from extended travel. As a result this individual's SSS values were abnormally high (ranging from 5 "fighting sleep" to 8 "being asleep"). Becaise this subject was also absent on day 1 and did not receive the relaxation intervention, and because extreme sleepiness may mask any specific effects of acupressure, the final analysis excluded this individual. To investi- 
gate the influence of this subject on the study results, an exploratory analysis was performed with inclusion of this outlier.

\section{RESULTS}

\section{Between-sequence covariate comparisons}

Thirty-nine (39) subjects were randomized into two acupressure treatment arms: 18 to Sequence I (stimulation-relaxation-relaxation) and 21 to Sequence II (relaxation-stimulation-stimulation). There were no differences in gender (I:9 male versus II:12 male; $\chi^{2}=0.199 ; p=0.656$ ) or ethnicity (I: 14 white versus II: 12 white; $\chi^{2}=1.857 ; p=0.173$ ) between the two sequences. The distribution of baseline categorical and continuous variables by sequence, treatment, and day are depicted in Table 1. No significant differences were observed for day 1 between treatment groups. Important covariates measured during the study, including com- pliance, are also presented in Table 1. After treatment on day 1 , more subjects in the relaxation acupressure group took naps $(p=0.048)$ and more participants in the stimulation group consumed caffeine $(p=0.039)$. However, there were no differences between the two groups in the quantity of caffeine consumed $(p=0.317)$.

\section{Analysis}

A mixed-model regression analysis using the change in SSS as the dependent variable was performed with data excluding the influential outlier. The following variables were retained from the full model: treatment $(p=0.019)$, day $(p=0.004)$, morning caffeine $(p=0.083)$, hours of overnight sleep $(p=$ 0.042 ), and morning upsetting event ( $p=0.071)$ (Table 2). No significant carry over effects between days or treatments were detected. The least-square means for the stimulation and relaxation acupressure treatments were 0.570 and 1.127 , respectively, with a significant difference in change in alertness scores between the two acupressure treatments.

Table 1. Covariates by Day, Sequence, and Treatment Assignment*

\begin{tabular}{|c|c|c|c|c|c|c|c|}
\hline & \multicolumn{3}{|c|}{ Day 1} & \multicolumn{2}{|c|}{ Day 2} & \multicolumn{2}{|c|}{ Day 3} \\
\hline & $I I$ & $I$ & & $I$ & $I I$ & $I$ & $I I$ \\
\hline & Relaxation & Stimulation & $\mathrm{p}$ & Relaxation & Stimulation & Relaxation & Stimulation \\
\hline \multicolumn{8}{|l|}{ Before acupressure } \\
\hline \multicolumn{8}{|l|}{ Categorical } \\
\hline AM caffeine & $14(70)$ & $13(72)$ & 0.880 & $16(89)$ & $15(75)$ & $13(76)$ & $12(60)$ \\
\hline AM upsetting event & $10(50)$ & $11(61)$ & 0.492 & $8(44)$ & $4(20)$ & $3(17.7)$ & $1(5)$ \\
\hline AM unusual event & $3(15)$ & $3(16.7)$ & $1.00^{\mathrm{a}}$ & $2(11)$ & $5(25)$ & $3(17.6)$ & $4(15)$ \\
\hline AM nap & 0 & 0 & $N A$ & 0 & $1(5)$ & 0 & $1(5)$ \\
\hline AM alcohol & $3(15)$ & $0(0)$ & $0.232^{\mathrm{a}}$ & $3(17)$ & $0(0)$ & $1(5.9)$ & $2(10)$ \\
\hline $\begin{array}{l}\text { Medication the evening } \\
\text { before }\end{array}$ & $9(45)$ & $4(22)$ & 0.140 & $6(33)$ & $7(35)$ & $6(35)$ & $7(35)$ \\
\hline \multicolumn{8}{|l|}{ Continuous } \\
\hline AM caffeine $(\mathrm{oz})$ & $8.4(7.3)$ & $12.4(11.9)$ & 0.231 & $11.3(8.4)$ & $9.0(9.0)$ & $10.2(8.2)$ & $9.7(13.9)$ \\
\hline $\begin{array}{l}\text { Hours of sleep the } \\
\text { night before }\end{array}$ & $5.4(1.1)$ & $5.5(1.5)$ & 0.821 & $5.9(1.4)$ & $6.0(1.1)$ & $6.8(1.4)$ & $6.8(0.80)$ \\
\hline Quality of sleep ${ }^{b}$ & $2.1(1.0)$ & $2.2(1.0)$ & 0.839 & $2.4(1.1)$ & $2.3(0.9)$ & $1.8(0.8)$ & $1.7(0.8)$ \\
\hline \multicolumn{8}{|l|}{ After acupressure } \\
\hline \multicolumn{8}{|l|}{ Categorical } \\
\hline Acupressure interference & $1(5)$ & $1(5.6)$ & $1.00^{\mathrm{a}}$ & $2(11)$ & $2(10)$ & $1(5.9)$ & $2(10)$ \\
\hline PM nap & $5(25)$ & $0(0)$ & $0.048^{\mathrm{a}}$ & $1(5.6)$ & $3(15)$ & $1(5.9)$ & $1(5)$ \\
\hline PM caffeine & $9(45)$ & $14(78)$ & 0.039 & $12(67)$ & $7(35)$ & $11(65)$ & $8(40)$ \\
\hline PM upsetting event & $6(30)$ & $8(44)$ & 0.357 & $12(67)$ & $9(45)$ & $3(17.7)$ & $1(5)$ \\
\hline PM out of ordinary event & $4(20)$ & $1(5.6)$ & $0.344^{\mathrm{a}}$ & $6(33)$ & $5(25)$ & $1(5.9)$ & $2(10)$ \\
\hline \multicolumn{8}{|l|}{ Continuous } \\
\hline PM caffeine (oz) & $7.6(10.3)$ & $10.6(7.2)$ & 0.317 & $7.7(6.4)$ & $6.1(9.5)$ & $10.7(9.1)$ & $6.8(10.2)$ \\
\hline Acupressure minutes & $14.8(0.5)$ & $15(0)$ & 0.187 & $14.3(2.4)$ & $14.9(0.4)$ & $15(0)$ & $15(0)$ \\
\hline Confident applied well ${ }^{\mathrm{c}}$ & $79.8(12.9)$ & $71.7(17.0)$ & 0.106 & $73.9(20.9)$ & $65.2(20.6)$ & $76.1(17.7)$ & $73.7(17.8)$ \\
\hline
\end{tabular}

Categorical variables [count (\%)] and continuous [mean (standard deviation)] covariates by day, sequence, and treatment assignment ( $p$ values were calculated only for day 1 because treatment effects could affect differences on days 2 and 3 .)

* Outlier removed from data.

aFisher's exact test.

${ }^{\mathrm{b}}$ Five-point Likert scale $(1=\operatorname{good}, 2=$ pretty good; $3=$ fair; $4=$ poor; $5=$ bad $)$.

${ }^{\mathrm{c}}$ Visual analog scale $(0=$ not confident; $100=$ very confident $)$. 
Table 2. Results of Mixed Regression Models

\begin{tabular}{|c|c|c|c|c|}
\hline & & Estimate & $S E$ & $\mathrm{p}$-value \\
\hline \multicolumn{5}{|l|}{ Final model } \\
\hline Treatment $^{\mathrm{a}}$ & & 0.557 & 0.232 & 0.019 \\
\hline \multirow[t]{3}{*}{ Day $^{b}$} & 1 & 0.753 & 0.337 & 0.029 \\
\hline & 2 & -0.256 & 0.307 & 0.407 \\
\hline & (reference) 3 & - & - & - \\
\hline AM Caffeine (oz) & & -0.022 & 0.012 & 0.083 \\
\hline Hours of sleep night before & & 0.208 & 0.100 & 0.042 \\
\hline Pre upsetting event & & 0.521 & 0.283 & 0.071 \\
\hline \multicolumn{5}{|c|}{ Final model with masking assessed } \\
\hline Blinding & & -0.255 & 0.245 & 0.303 \\
\hline Treatment & & 0.483 & 0.240 & 0.048 \\
\hline \multirow[t]{3}{*}{ Day $^{\mathrm{c}}$} & 1 & 0.708 & 0.339 & 0.041 \\
\hline & 2 & -0.275 & 0.308 & 0.376 \\
\hline & (reference) 3 & - & - & - \\
\hline AM Caffeine (oz) & & -0.022 & 0.012 & 0.077 \\
\hline Hours of sleep night before & & 0.205 & 0.101 & 0.046 \\
\hline Pre upsetting event & & 0.515 & 0.284 & 0.075 \\
\hline
\end{tabular}

an this model, if AM caffeine and pre upsetting events are removed, the treatment estimate (SE [standard error]) was $0.523(0.230)$ with a $p$-value of 0.0264 .

b In this model, the $p$-value for the overall fixed effect of day was 0.004 .

'In this model, the $p$-value for the overall fixed effect of day was 0.006 .

The mean difference between morning and afternoon SSS scores are presented by treatment, sequence, and day in Figure 2. The relaxation acupressure treatments consistently produced higher values, corresponding to more fatigue than the stimulation acupressure treatments regardless of sequence or day.

In an exploratory analysis to examine the effect of the outlier's responses on the study results, the above model was fit with data from the outlier included. The significance of treatment $(p=0.077)$, day $(p=0.015)$, hours of overnight

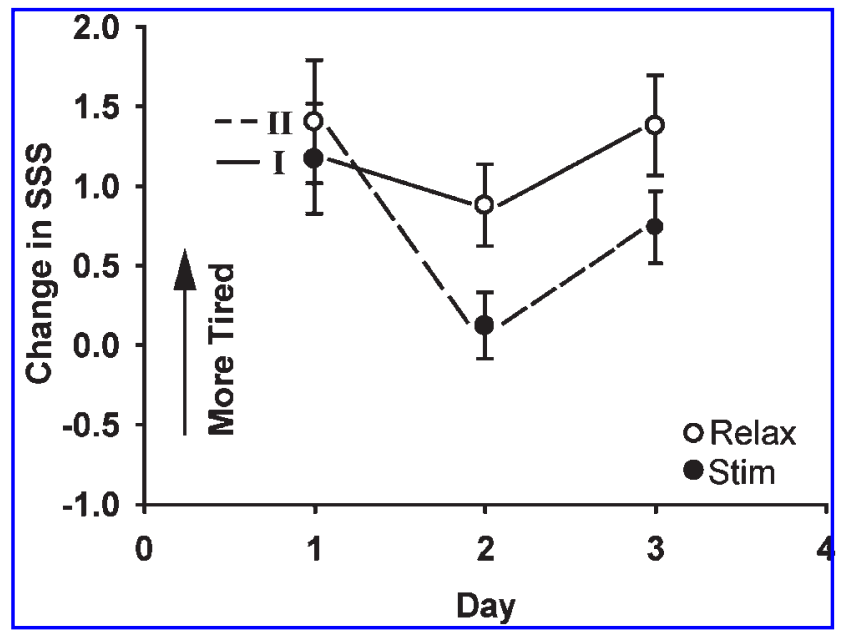

FIG. 2. Plot of mean (standard error) change in Stanford Sleepiness Scale (SSS) (afternoon-morning) scores by sequence and day indicated a significant treatment effect in the final model $(p=$ 0.0194). Solid symbols indicate stimulation treatment; open symbols, relaxation treatment. sleep $(p=0.069)$, and morning caffeine intake $(p=0.122)$ were changed by this inclusion. In this model, the adjusted least square means for the stimulation and relaxation treatments were 0.736 and 1.196 , respectively, with a trend toward less fatigue with stimulation acupressure.

\section{Compliance, masking, and adverse effects}

Reported compliance in the study for both acupressure arms was excellent (>90\%), and no significant differences between sequences were found.

Masking was assessed by questionnaire after treatment day 1 yielding a continuity-corrected $\chi^{2}$ test result of $\chi^{2}=$ $2.63, p=0.104)$, suggesting that blinding was imperfect. Twenty-five (25) of 38 participants were able to guess their treatment (relaxation or stimulation) correctly on day 1 . Over the course of the trial, $62 \%$ of participants were able to discern their treatment allocation. To evaluate the significance of the effect of masking on the outcome of the trial, the subjects' assessments of which treatment arm they were assigned were included as covariates in the model with the outlier excluded (Table 2). Although inclusion of masking increased the $p$ value from 0.0194 to 0.0484 , the treatment effect remained statistically significant in this model as well. The $p$ values of the other covariates were slightly changed: day $(p=0.006)$, hours of overnight sleep $(p=0.046)$, morning caffeine intake $(p=0.077)$, and upsetting event in the morning $(p=0.075)$. In this model, the adjusted least square means for the stimulation and relaxation treatments were 0.614 and 1.100 , respectively, with significantly less fatigue reported with stimulation acupressure. 
Few side-effects were reported. Muscle cramps $(n=5)$, muscle aches $(n=5)$, headaches $(n=6)$, and fatigue $(n=$ 7) were the most common side-effects. All were of brief duration and were neither clinically significant for individuals nor statistically significantly different between sequences.

\section{DISCUSSION}

This study assessed the effects of two forms of acupressure on the change in alertness in a population of healthy medical professionals in a prolonged lecture situation. It was hypothesized that the two acupressure regimens would differ in their impact on the change in alertness throughout the day after controlling for covariates. This study showed that a stimulation acupressure regimen has a statistically significant reduction in sleepiness, as measured by SSS, compared to a relaxation acupressure treatment. This finding suggests that acupressure can change alertness in individuals who are in classroom settings for a full day.

The use of a cross-over design allowed subjects to serve their own controls, thereby reducing potential confounders. The use of two treatment regimens that had opposing consequences for alertness may have simultaneously increased the magnitude of the treatment effect and removed the problem of noninert sham acupressure treatments present in previous studies. $^{8-10}$ The self-administered acupressure treatments were learned easily by the subjects, suggesting that this methodology may be easily disseminated to other subjects with no prior knowledge of acupressure theory or techniques.

However, several limitations were inherent to the study. Although it was a single-blind, randomized design, the majority of study subjects were able to discern their treatment arms, thereby posing a potential bias in the study's primary outcome findings. This problem may have been in part caused by the use of tapping of the Shi Shen Chong site on the top of the head in the stimulation acupressure regimen, which may have been difficult to interpret as relaxing. Despite this weakness, a significant effect of acupressure treatment was still observed when the subjects' assessments of their treatment assignments were incorporated into the model.

A second limitation lies in the potential for generalization of the study findings to the general population. The participants are well educated, are in academic teaching positions, and are active scientific researchers. Moreover, the study was conducted in a clinical trial design class in which the participants were motivated to learn and also compliant. As a result, it remains unclear whether the general population would be as motivated to learn the acupressure techniques or equally compliant with the treatment regimen as was this study population. Furthermore, the results should not be extrapolated to severely sleepy individuals, as evidenced by the effect of including the outlier in our analysis.

A third limitation to the study involves the design of the stimulation and relaxation acupressure arms. Because no ac- cepted stimulation or relaxation acupressure interventions exist, the acupressure sequences used for each treatment were empirically developed. It remains unclear whether these combinations of five points in each acupressure treatment arm are the optimal sites and whether 3 minutes each is the optimal treatment duration. It is quite possible that lesser or greater pressure, duration, or different locations of acupressure may be significantly more effective. In addition, the acupuncture points and formulas implemented here should not be used indiscriminately. Different acupressure techniques used on the same point may have differing outcomes in the hands of trained acupuncturists, a factor not addressed in this investigation.

Although this study showed a statistically significant difference in change in SSS scores between the stimulation and relaxation acupressure arms, it is unclear whether this difference corresponds to a meaningful difference in subject performance using objective outcomes. For instance, it is not clear that a mean difference in change in SSS scores of 0.56 [1.127 (relaxation) -0.570 (stimulation) $]$ means that stimulatory acupressure will enable students to perform better in classroom settings.

This investigation also raises a potential ethical issue. Participant performance in class may have been compromised by the sedation acupressure. Although the sedating effects of acupressure were not dramatic, future trials may wish to implement a mental alertness task, the results of which would not make a direct impact on the participants.

Finally, because the two treatment arms for comparison were a relaxation arm and a stimulation arm, it is not clear whether stimulation acupressure decreased fatigue, whether relaxation acupressure increased fatigue, or whether both simultaneously occurred. One can only conclude that there was a difference between treatments.

\section{CONCLUSIONS}

In summary, this study showed that a stimulation acupressure regimen significantly decreased fatigue and improved alertness in a classroom setting compared to a relaxation acupressure regimen. This significant difference between two acupressure regimens on subjects' perceived fatigue suggests that acupressure may have effects on human alertness. Further studies are required in other populations to determine the scope of this effect, to optimize these acupressure regimens, and to measure performance outcomes to determine whether acupressure has an impact not only on perceived fatigue but also on human function.

\section{ACKNOWLEDGMENTS}

The authors thank Dr. Sara Warber and members of the University of Michigan Integrative Medicine program for 
the idea of performing a clinical trial within a classroom setting. Thanks are also expressed to the instructors of the University of Michigan Clinical Research Design and Statistical Analysis Program for consultation, revision, and approval of this work. R.E.H. was supported by National Institutes of Health (NCCAM) K01 AT01111-01.

\section{REFERENCES}

1. Owens JA. Sleep loss and fatigue in medical training. Curr Opin Pulmon Med 2001;7;411-418.

2. Bonnet MH, Arand DL. We are chronically sleep deprived. Sleep 1995;18:908-911.

3. Akerstedt T, Ficca G. Alertness-enhancing drugs as a countermeasure to fatigue in irregular work hours. Chronobiol Int 1997;14:145-158.

4. Caldwell JA Jr. Fatigue in the aviation environment: An overview of the causes and effects as well as recommended countermeasures. Aviat Space Environ Med 1997;68:932-938.

5. Giam GC. Effects of sleep deprivation with reference to military operations. Ann Acad Med Singapore 1997;26: 88-93.

6. Bonnefond A, Tassi P, Roge J, Muzet A. A critical review of techniques aiming at enhancing and sustaining workers alertness during the night shift. Ind Health 2004;42:1-14.

7. Lin Y. Acupuncture treatment for insomnia and acupuncture analgesia. Psychiatry Clin Neurosci 1995;49:119-120.
8. Tsay SL, Rong JR, Lin PF. Acupoints massage in improving the quality of sleep and quality of life in patients with endstage renal disease. J Advanc Nurs 2003;42:134-142.

9. Tsay SL, Chen ML. Acupressure and quality of sleep in patients with end-stage renal disease-a randomized controlled trial. Int J Nurs Studies 2003;40:1-7.

10. Tsay SL. Acupressure and fatigue in patients with end-stage renal disease - a randomized controlled trial. Int J Nurs Studies 2004;41:99-106.

11. Xinnong C. Chinese Acupuncture and Moxibustion. Bejing: Foreign Languages Press, 1999.

12. Chu Z. Fundamentals of Chinese Medicine [Wiseman N, Ellis A, transl]. Brookline, MA: Paradigm Publications, 1996.

13. Ross J. Acupuncture Point Combinations: The Key to Clinical Success. New York: Churchill Livingstone, 1998.

14. Hoddes E, Zarcone V, Smythe H, et al. Quantification of sleepiness: A new approach. Psychophysiology 1973;10:431-436.

Address reprint requests to:

Richard E. Harris, Ph.D.

Department of Internal Medicine

Division of Rheumatology

University of Michigan

Chronic Pain and Fatigue Research Center

24 Frank Lloyd Wright Drive, P.O. Box 385

Ann Arbor, MI 48106

E-mail: reharris@med.umich.edu 